\title{
Tightly Coupled UWB/IMU Pose Estimation
}

\author{
Jeroen D. Hol*†, Fred Dijkstra*, Henk Luinge* and Thomas B. Schön ${ }^{\dagger}$ \\ ${ }^{*}$ Xsens Technologies B.V., Enschede, The Netherlands \\ $\dagger$ Division of Automatic Control, Linköping University, Sweden
}

\begin{abstract}
In this paper we propose a $6 \mathrm{DOF}$ tracking system combining Ultra-Wideband measurements with low-cost MEMS inertial measurements. A tightly coupled system is developed which estimates position as well as orientation of the sensorunit while being reliable in case of multipath effects and NLOS conditions. The experimental results show robust and continuous tracking in a realistic indoor positioning scenario.
\end{abstract}

\section{INTRODUCTION}

Ultra-wideband (UWB) is a relatively new and promising localization technology, especially for indoor applications. Among its more mature applications are the so-called asset tracking systems in for instance health-care or manufacturing. Commercially available systems [1,2] typically consist of a network of synchronized UWB receivers which track a large number of small, battery powered and inexpensive UWB transmitters. Reported indoor position accuracies lie in the order of decimeters, but suffer from multipath effects and non-line-of-sight (NLOS) conditions. These effects are most prominent while tracking moving objects or persons and give rise to distorted and bumpy trajectories. Although the obtained performance is often sufficient for the aforementioned applications, many potential application areas have higher performance requirements.

To improve the tracking performance (especially the positioning accuracy) we propose to combine UWB with a low-cost micro electro mechanical system (MEMS) inertial measurement unit (IMU) consisting of a 3D rate gyroscope and a 3D accelerometer. The main justification for adding an IMU — providing accurate position tracking for short periods of time, but drift prone for longer timescales — is to obtain a robust system, capable of detecting and rejecting multipath effects and NLOS situations. Additional benefits of adding an IMU include improved tracking results, especially for dynamic quantities like velocity, and that the orientation becomes observable as well. This results in a system providing a 6 degrees of freedom (DOF) general purpose tracking solution for indoor applications.

To the best of the authors' knowledge there are only a few reports in the literature on combining UWB and inertial sensors. The more recent contributions include a hybrid 2D positioning tracking algorithm [3] and an EKF for pedestrian tracking [4]. Both approaches are loosely coupled and only estimate a limited number of DOF. By a loosely coupled approach we refer to a solution where the measurements from one or several of the individual sensors are preprocessed before they are used to compute the final result. A tightly coupled approach on the other hand refers to an approach where all the measurements are used directly to compute the final result. In this paper we propose a full $6 \mathrm{DOF}$ tracker estimating both

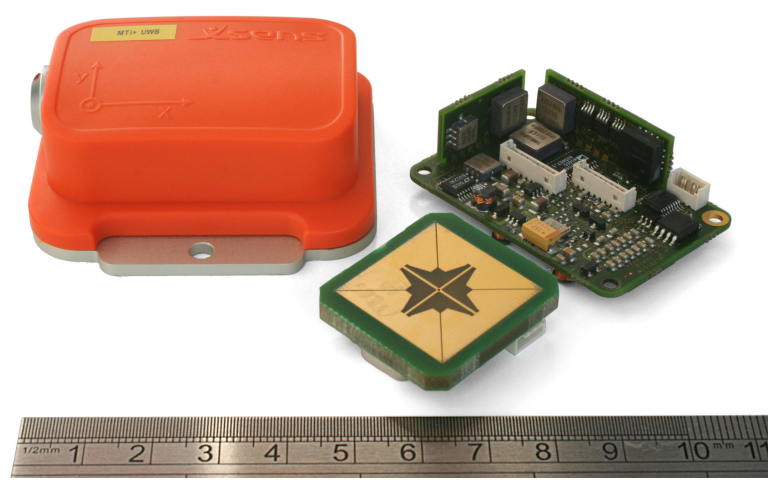

Fig. 1. The sensor unit, integrating an IMU and an UWB transmitter into a single housing.

position and orientation based on tightly coupled fusion of UWB and inertial sensors. However, note that in order to obtain heading (i.e., the angle around the vertical) observability, there has to be some amount of acceleration present.

\section{PRoblem Formulation}

In this section we will give a more detailed formulation of the tracking problem we are trying to solve. We start by properly introducing the setup that is used.

Our setup is based on a commercially available asset tracking system. However, instead of working with an UWB transmitter only, we integrated it with an IMU in a single unit, shown in Fig. 1. The devices are synchronized at hardware level, significantly simplifying the signal processing. The pulses transmitted by the sensor unit are detected by a network of UWB receivers with synchronized clocks.

This setup gives rise to the following coordinate frames, illustrated in Fig. 2.

- Navigation frame (n): The sensor unit position and orientation (pose) is estimated with respect to this stationary coordinate frame. It can be defined arbitrarily, here it is aligned with the room, with the vertical axis pointing up. The UWB receiver positions are known in this frame and are, without loss of generality, assumed to be constant.

- Body frame (b): This is the coordinate frame of the moving IMU. Its origin is located in the center of the accelerometer axes, and it is aligned to the casing. All the inertial measurements are resolved in this coordinate frame.

These coordinate frames are used to express quantities in as well as to denote their origin. For instance, $\boldsymbol{b}^{n}$ is the position of the body coordinate frame expressed in the navigation frame 


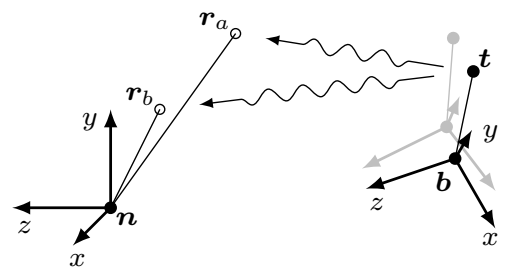

Fig. 2. The sensor unit, shown at two time instants, consists of an IMU (b) and a UWB transmitter $(\boldsymbol{t})$. Transmitted signals are picked up by the UWB receivers $(\boldsymbol{r})$ in the navigation $(\boldsymbol{n})$ frame.

and $\boldsymbol{q}^{b n}, \boldsymbol{\varphi}^{b n}, \boldsymbol{R}^{b n}$ are the unit quaternion, the rotation vector and the rotation matrix, respectively, all interchangeable and describing the rotation from the navigation frame to the body frame. A good overview of various rotation parameterizations is given in [5]. Other quantities of interest are the positions of the UWB transmitter and receivers, denoted $\boldsymbol{t}$ and $\boldsymbol{r}_{m}$, respectively. The UWB transmitter and the IMU are rigidly connected, i.e., $\boldsymbol{t}^{b}$ is a known constant.

The objective of this work is to develop a method to track the position and orientation of the sensor unit, i.e., to estimate

- The position of the body expressed in the navigation frame, $\boldsymbol{b}^{n}$.

- The orientation of the body with respect to the navigation frame, $\boldsymbol{q}^{b n}$.

In order to estimate these quantities tightly coupled sensor fusion is used, illustrated by Fig. 3. That is, the 'raw' sensor

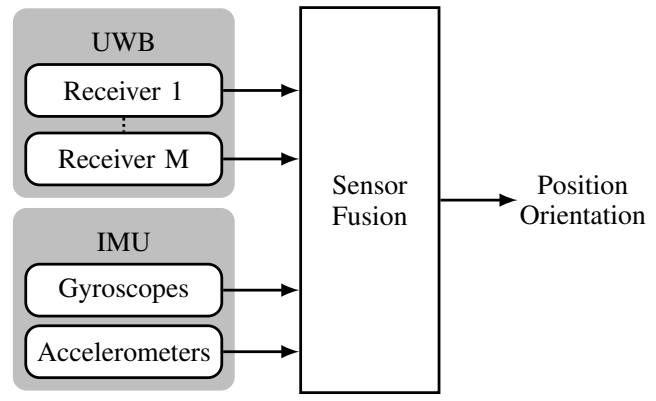

Fig. 3. Tightly coupled sensor fusion. The 'raw' measurements from the $M$ UWB receivers and the IMU are directly used for sensor fusion.

measurements - measurements from the sensing components such as accelerometer, gyroscope and time of arrival (TOA) measurements - are directly used for sensor fusion, instead of already filtered output quantities like position or acceleration. Hence, there is no explicit triangulation module as typically found in (loosely coupled) UWB positioning systems. Instead, the triangulation of position is implicitly performed by the sensor fusion algorithm.

The advantages of using a tightly coupled approach are twofold. Firstly, preprocessing of measurements typically results in loss of information. This is mainly due to approximations of statistical distributions, but in extreme cases measurements are ignored, for instance when there are not enough TOA measurements for triangulation. By directly using the sensor measurements nothing has to be disregarded and maximal advantage is taken of the available information. Secondly, tightly coupled sensor fusion can perform hypothesis testing for the individual sensors and efficiently deal with outliers [6]. This is especially useful for UWB measurements, where outliers occur regularly due to multipath effects and/or NLOS conditions. Tightly coupled sensor fusion can disregard the affected measurements while still utilizing the remaining ones. Additionally, the available inertial information gives accurate predictions of the UWB measurements, which allows for improved outlier detection. Hence, a tightly coupled system is more robust.

The basic ingredient of any sensor fusion method is a state space model of the underlying process. This is the topic of the next section.

\section{MODELING}

The sensor fusion approach briefly introduced in the previous section requires a model of the sensor unit. We aim to provide a thorough background and start with an analysis of inertial and UWB sensors in Section III-A and Section III-B, respectively. Together with the dynamics, discussed in Section III-C, these models form the basis for an Extended Kalman Filter (EKF) in Section III-D.

\section{A. Inertial sensors}

An inertial measurement unit consists of accelerometers and rate gyroscopes. The gyroscopes measure angular velocity or rate-of-turn $\boldsymbol{\omega}$. The accelerometers do not measure accelerations directly, but rather the so-called external specific force $f$ to which the linear acceleration $\ddot{b}$ as well as the earth's gravitational field $\boldsymbol{g}$ contribute.

The measurements from the accelerometers and gyroscopes can be used to compute the position and orientation of an object relative to a known starting point using inertial navigation [7-9]. In a strap-down configuration such as our sensor unit, the measurements are resolved in the body coordinate frame, rather than in an inertial reference frame. Hence, the orientation $\boldsymbol{q}^{n b}$ can be calculated by integrating the angular velocity $\boldsymbol{\omega}_{n b}^{b}$. The position $\boldsymbol{b}^{n}$ can be obtained by double integration of the acceleration $\ddot{b}^{n}$, which in turn is found by rotating the external specific force $\boldsymbol{f}^{b}$ using the known orientation $\boldsymbol{q}^{n b}$ and subtracting the acceleration due to gravity. This procedure is illustrated in Fig. 4.

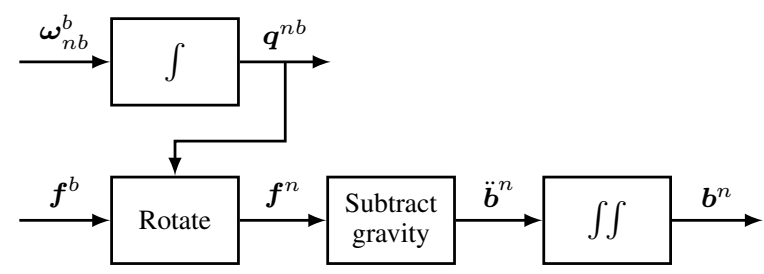

Fig. 4. Schematic of a strap-down inertial navigation algorithm.

The angular velocity $\boldsymbol{\omega}_{n b}^{b}$ and the external specific force $\boldsymbol{f}^{b}$ are measured by the gyroscope and the accelerometer. These measurements include bias and noise terms which cause errors in the calculated position and orientation. This integration drift is inherent to all inertial navigation. Using MEMS inertial sensors, the integration drift is relatively large. Hence, the orientation estimate and especially the position estimate, are 
only accurate and reliable for a short period of time. This is the reason why MEMS IMU's are typically used in combination with a stabilizing sensor such as GPS, vision or UWB.

Summarizing the above discussion, the gyroscope measurements are modeled as

$$
\boldsymbol{u}_{\omega}=\boldsymbol{\omega}_{n b}^{b}+\boldsymbol{\delta}_{\omega}^{b}+\boldsymbol{e}_{\omega}^{b} .
$$

Here, $\boldsymbol{\omega}_{n b}^{b}$ is the angular velocity, body to navigation, expressed in the body frame, $\delta_{\omega}^{b}$ is a slowly time-varying bias term and $\boldsymbol{e}_{\omega}^{b}$ is i.i.d. Gaussian noise. Furthermore, the accelerometer measurements are modeled as

$$
\boldsymbol{u}_{a}=\boldsymbol{f}^{b}+\boldsymbol{\delta}_{a}^{b}+\boldsymbol{e}_{a}^{b}=\boldsymbol{R}^{b n}\left(\ddot{\boldsymbol{b}}^{n}-\boldsymbol{g}^{n}\right)+\boldsymbol{\delta}_{a}^{b}+\boldsymbol{e}_{a}^{b},
$$

where $f^{b}$ is the external specific force expressed in the body coordinate system, $\boldsymbol{\delta}_{a}^{b}$ is a slowly time-varying bias and $\boldsymbol{e}_{a}^{b}$ is i.i.d. Gaussian noise. The second expression in (2) splits the specific force into its contributions from the linear acceleration of the sensor $\ddot{\boldsymbol{b}}^{n}$ and the gravity vector $\boldsymbol{g}^{n}$, both expressed in the navigation frame. These vectors have been rotated to the body frame using the rotation matrix $\boldsymbol{R}^{b n}$.

\section{B. Ultra-wideband}

Ultra-wideband technology makes use of radio with very short pulses, typically $\approx 1 \mathrm{~ns}$, resulting in a very high spatial resolution. The positioning technologies can be roughly subdivided into three categories: systems using time delay, systems using angle-of-arrival and systems using signal strength [10]. In this paper we focus on time based methods, where position is inferred from the time it takes for a signal to travel from the transmitter to the receiver.

The UWB setup consists of a network of synchronized UWB receivers, all taking very precise TOA measurements of signals originating from the transmitter in the sensor unit. That is, the measurement of the $m$-th receiver is given by

$$
y_{m}=\tau+\left\|\boldsymbol{r}_{m}^{n}-\boldsymbol{t}^{n}\right\|_{2}+e_{u, m},
$$

where $\tau$ is the time of transmission, $\boldsymbol{r}_{m}^{n}$ is the position of the $m$-th receiver in the navigation frame, $\boldsymbol{t}^{n}$ is the position of transmitter in the navigation frame and $e_{u, m}$ is i.i.d. Gaussian noise. All quantities in (3) are expressed in meters. The transmitter clock is not accurate enough to know the precise time of transmission $\tau$, and since only one-way communication is possible it is not synchronized to the receiver clocks. Therefore, the time of transmission $\tau$ has to be treated as an unknown. This makes the UWB measurements very similar to GPS pseudo-ranges [11].

The process of determining the transmitter position from the UWB measurements is referred to as triangulation, for which several approaches can be found in literature. A common technique is to eliminate the time of transmission $\tau$ from (3) by constructing time difference of arrival (TDOA) measurements from pairs of measurements. The resulting set of hyperbolic equations can then be efficiently solved for position [12, 13]. The drawback of this approach is that the constructed TDOA measurements are no longer independently distributed. An alternatively triangulation method is to treat $\tau$ as an unknown and solve for position and time. Assuming Gaussian noise, maximum likelihood estimation takes the form of a nonlinear least squares problem,

$$
\min _{t^{n}, \tau} \sum_{m=1}^{M}\left(y_{m}-\tau-\left\|\boldsymbol{r}_{m}^{n}-\boldsymbol{t}^{n}\right\|_{2}\right)^{2},
$$

which can be efficiently solved [14].

\section{Dynamics}

The inertial and UWB measurement models are linked by a process model, which describes the motion of the sensor unit. Since it is hard to make informative assumptions regarding general sensor unit movement, the inertial sensors are used as inputs $\boldsymbol{u}_{t}$ for the process model rather than treating them as measurements. Following the derivation in [15], we have

$$
\begin{aligned}
& \boldsymbol{b}_{t+1}^{n}=\boldsymbol{b}_{t}^{n}+T \dot{\boldsymbol{b}}_{t}^{n}+\frac{T^{2}}{2} \ddot{\boldsymbol{b}}_{t}^{n}, \\
& \dot{\boldsymbol{b}}_{t+1}^{n}=\dot{\boldsymbol{b}}_{t}^{n}+T \ddot{\boldsymbol{b}}_{t}^{n}, \\
& \boldsymbol{q}_{t+1}^{b n}=e^{-\frac{T}{2} \boldsymbol{\omega}_{n b, t}^{b} \odot \boldsymbol{q}_{t}^{b n},}
\end{aligned}
$$

where $\boldsymbol{b}^{n}$ and $\dot{b}^{n}$ denote the position and velocity of the body resolved in the navigation frame, $\boldsymbol{q}^{b n}$ is a unit quaternion describing the orientation of the body frame relative to the navigation frame and $T$ denotes the sampling interval. Furthermore, $\odot$ is the quaternion multiplication and the quaternion exponential is defined as

$$
e^{(0, \boldsymbol{v})} \triangleq\left(\cos \|\boldsymbol{v}\|, \frac{\boldsymbol{v}}{\|\boldsymbol{v}\|} \sin \|\boldsymbol{v}\|\right) .
$$

The acceleration $\ddot{\boldsymbol{b}}_{t}^{n}$ and angular velocity $\boldsymbol{\omega}_{n b, t}^{b}$ are calculated from the accelerometer measurement $\boldsymbol{u}_{a}$ and the gyroscope measurement $\boldsymbol{u}_{\omega}$ according to

$$
\begin{aligned}
\ddot{\boldsymbol{b}}_{t}^{n} & =\boldsymbol{R}_{t}^{n b} \boldsymbol{u}_{a, t}+\boldsymbol{g}^{n}-\boldsymbol{R}_{t}^{n b} \boldsymbol{\delta}_{a}^{b}-\boldsymbol{R}_{t}^{n b} \boldsymbol{e}_{a, t}^{b}, \\
\boldsymbol{\omega}_{n b, t}^{b} & =\boldsymbol{u}_{\omega, t}-\boldsymbol{\delta}_{\omega}^{b}-\boldsymbol{e}_{\omega, t}^{b} .
\end{aligned}
$$

The inertial bias terms $\boldsymbol{\delta}_{a}^{b}$ and $\boldsymbol{\delta}_{\omega}^{b}$ are slowly time-varying. Hence, they are included in the process model as random walk, according to

$$
\begin{aligned}
& \boldsymbol{\delta}_{a, t+1}^{b}=\boldsymbol{\delta}_{a, t}^{b}+\boldsymbol{v}_{\delta_{a}, t}^{b}, \\
& \boldsymbol{\delta}_{\omega, t+1}^{b}=\boldsymbol{\delta}_{\omega, t}^{b}+\boldsymbol{v}_{\delta_{\omega}, t}^{b},
\end{aligned}
$$

where $\boldsymbol{v}_{\delta_{a}}^{b}, \boldsymbol{v}_{\delta_{\omega}}^{b}$ are i.i.d. Gaussian noises.

The time of transmission $\tau$ has to be included in the model as well. Since the transmitter sends in regular intervals, $\tau$ is modeled as an integrated random walk

$$
\begin{aligned}
& \tau_{t+1}=\tau_{t}+T \dot{\tau}_{t}+v_{\tau, t}, \\
& \dot{\tau}_{t+1}=\dot{\tau}_{t}+v_{\dot{\tau}, t},
\end{aligned}
$$

where $v_{\tau}, v_{\dot{\tau}}$ are i.i.d. Gaussian noises. Here, $v_{\tau}$ is used to model the jitter inherently present in the clock.

The UWB measurement model (3) requires the transmitter position $\boldsymbol{t}^{n}$. The sensor unit motion (4), however, is modeled using the pose of the body coordinate system $\boldsymbol{q}^{b n}, \boldsymbol{b}^{n}$. Hence, the relation

$$
\boldsymbol{t}_{t}^{n}=\boldsymbol{b}_{t}^{n}+\boldsymbol{R}^{n b} \boldsymbol{t}^{b}
$$

is used to calculate $t^{n}$. 


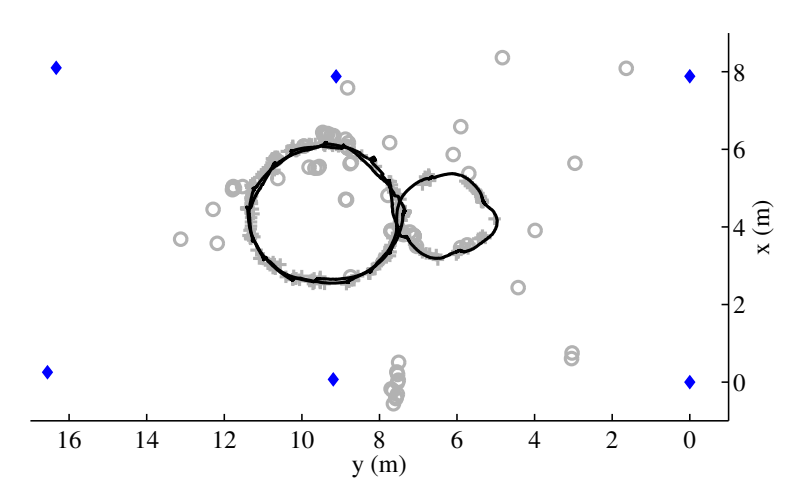

(a)

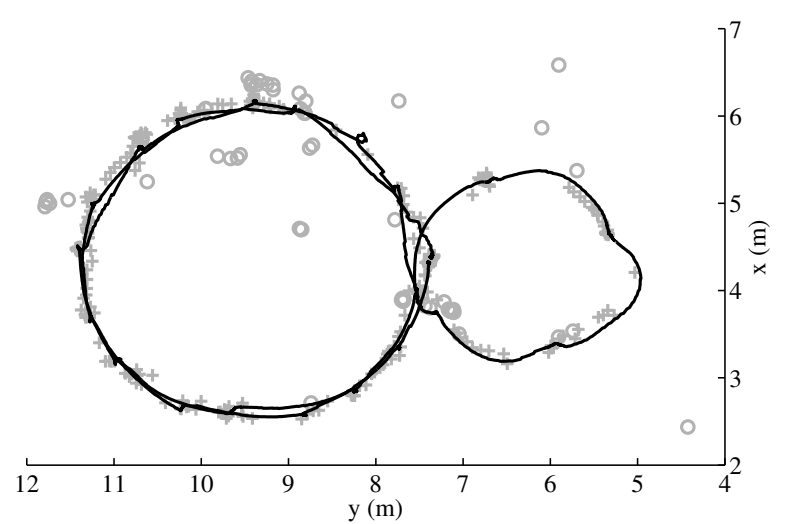

(b)

Fig. 5. Overview (a) and detail (b) of the trial with a test subject walking an eight-shaped trajectory. Shown are the estimated trajectory $\boldsymbol{b}^{n}$ (-), triangulated positions, classified according to whether the UWB measurements are clean $(+)$ or contain outliers $(\circ)$, and the UWB receivers $(\diamond)$. The tightly coupled approach successfully bridges the 'gaps' in the triangulated positions and is not affected by outliers.

\section{Sensor fusion}

Combining (3)-(9) we obtain a discrete-time nonlinear state-space model with state vector

$$
\boldsymbol{x}=\left(\left(\boldsymbol{b}^{n}\right)^{T},\left(\dot{\boldsymbol{b}}^{n}\right)^{T},\left(\boldsymbol{q}^{b n}\right)^{T},\left(\boldsymbol{\delta}_{a}^{b}\right)^{T},\left(\boldsymbol{\delta}_{\omega}^{b}\right)^{T}, \tau, \dot{\tau}\right)^{T} .
$$

In this paper, we use it in combination with an extended Kalman filter (EKF) [16] to fuse the TOA and inertial measurements. The EKF handles the different sample rates and a varying number of measurements straightforwardly. It runs at the high data rate of the IMU $(200 \mathrm{~Hz})$ and the $50 \mathrm{~Hz}$ UWB updates are only performed when measurements are available.

Outliers from NLOS and/or multipath effects are detected using hypothesis testing on the residuals/innovations of the EKF,

$$
\boldsymbol{\epsilon}_{t}=\boldsymbol{y}_{t}-\hat{\boldsymbol{y}}_{t \mid t-1}
$$

the difference between the observed measurement $\boldsymbol{y}_{t}$ and the one-step ahead prediction from the model $\hat{\boldsymbol{y}}_{t \mid t-1}$. In absence of errors, the residuals are normal distributed as

$$
\boldsymbol{\epsilon}_{t} \sim \mathcal{N}\left(0, \boldsymbol{C}_{t} \boldsymbol{P}_{t \mid t-1} \boldsymbol{C}_{t}^{T}+\boldsymbol{R}_{t}\right),
$$

where $\boldsymbol{P}_{t \mid t-1}$ denotes the state covariance, $\boldsymbol{C}_{t}$ denotes the measurement Jacobian and $\boldsymbol{R}_{t}$ denotes the covariance of the measurement noise. This allows the calculation of confidence intervals for the individual measurements and in case these are violated, the measurement is considered an outlier and is ignored.

\section{EXPERIMENTAL RESULTS}

The proposed system has been used to track a test subject walking around in a relatively large room, approximately $18 \times 8 \times 2.5 \mathrm{~m}$ in size. The room is equipped with 6 synchronized UWB receivers at known locations that are attached to the ceiling. The sensor unit has been mounted on a foot of the test subject, a position with relatively high dynamics. Regular occurring NLOS conditions due to occlusion by the body - a medium with a reduced speed of light — as well as multipath

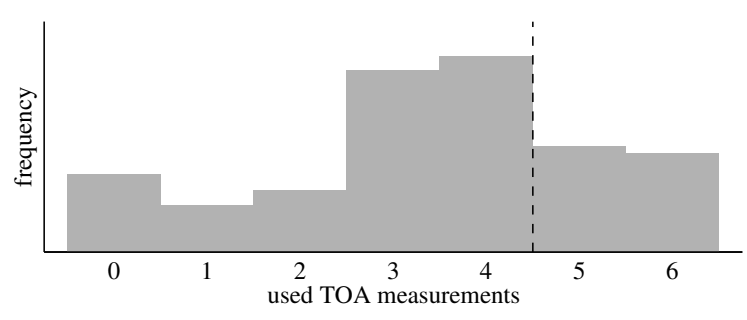

Fig. 6. Histogram of the number of TOA measurements used in the EKF after outlier rejection. Triangulation requires $\geq 5$ TOAs and is only limitedly possible.

effects from signals reflected by the floor result in difficulties during triangulation.

In this section we present the results for a $35 \mathrm{~s}$ trial where the test subject walked an eight-shaped path. Fig. 5 shows a top view of the estimated trajectory. Note that the triangulated positions (standalone UWB) contain many gaps as well as many outliers. In contrast, the proposed system is capable to estimate a continuous trajectory of the test subject. The tightly coupled fusion of UWB and inertial measurements makes it possible to make use of any number of UWB measurements and is hence capable to bridge the 'gaps' where not enough UWB measurements are available for 3D triangulation. Furthermore, the classification of the UWB solutions in Fig. 5 show that our approach successfully detects and deals with outliers in the UWB measurements.

The advantage gained by being able to utilize all available information is quantified in Fig. 6. Although in theory 4 TOA measurements are sufficient for a $3 \mathrm{D}$ position solution, in practice at least 5 measurements are required for successful triangulation of UWB measurements, implying that more than half of the available UWB measurements would have to be discarded. This results in prolonged periods without a position solution where also loosely coupled UWB inertial approaches are bound to fail.

The proposed system not only estimates the position of the sensor unit, but also provides very smooth orientation and velocity estimates, shown in Fig. 7 and Fig. 8. These are very 

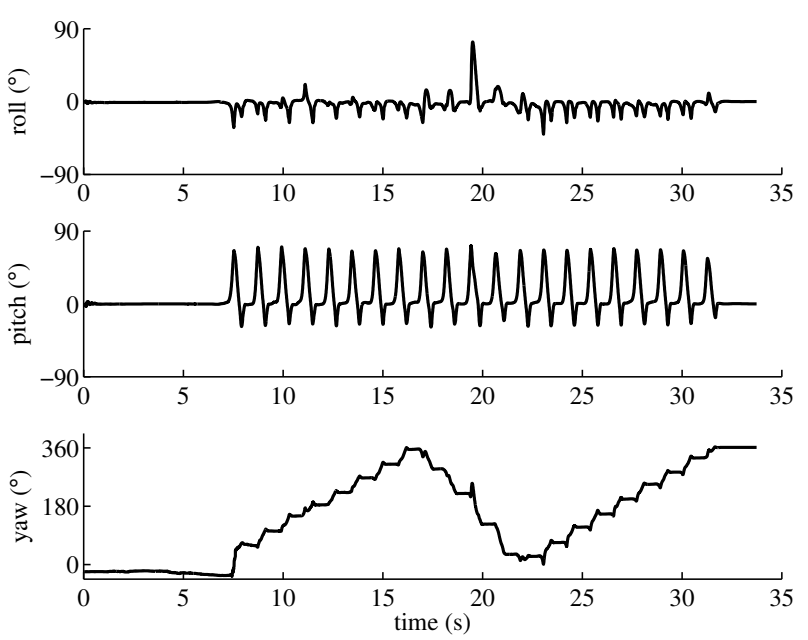

Fig. 7. Estimated orientation $\boldsymbol{q}^{n b}$, expressed in Euler angles.
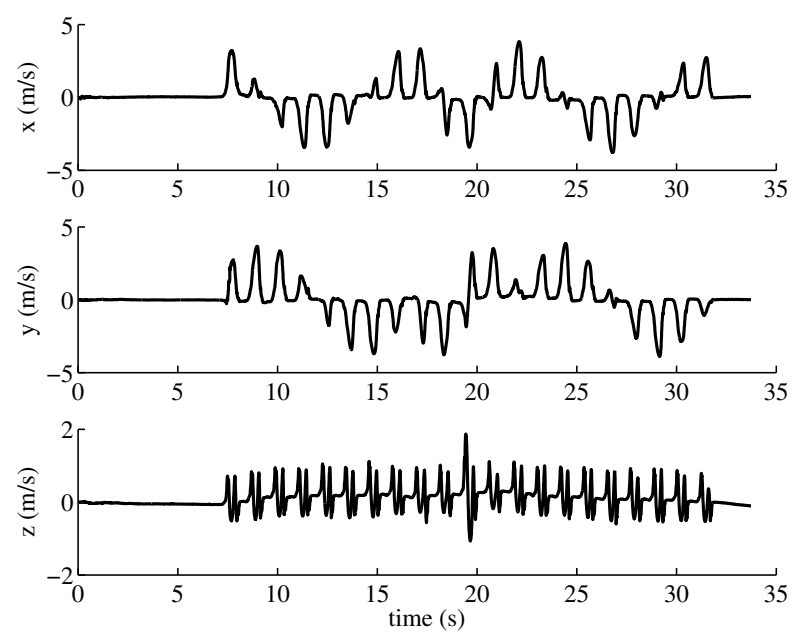

Fig. 8. Estimated velocity $\dot{b}^{n}$.

hard or impossible to obtain using standalone UWB systems.

The presented results show that tightly coupled fusion of UWB and inertial measurements results in accurate and robust tracking. However, the estimated height is not as accurate as can be expected, see Fig. 9. The test-subject walked on the floor, implying that heights close to $0 \mathrm{~m}$ are to be expected. Especially during motion the height variation of the UWB solution is larger what can be expected according to the dilution of precision (DOP). This could indicate that calibration errors are present in the UWB system.

\section{CONCLUSION}

In this paper a $6 \mathrm{DOF}$ tracking algorithm is proposed estimating both position and orientation based on tightly coupled fusion of UWB and inertial sensors. Experiments show that a robust and accurate system is obtained even in the presence of multipath and NLOS conditions. The system is capable to bridge periods with limited UWB measurements and successfully detects and deals with outliers in the individual TOA measurements.

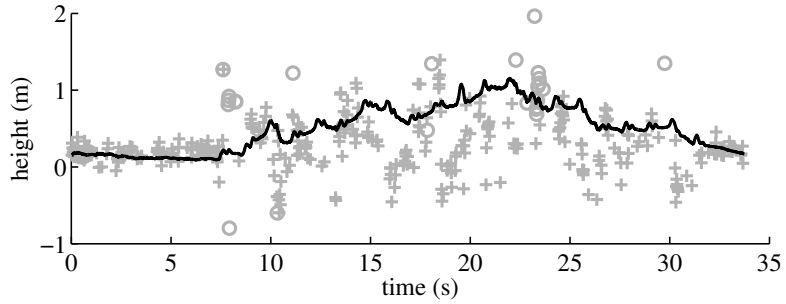

Fig. 9. Estimated height $\boldsymbol{b}_{z}^{n}$. Shown are the estimated trajectory (-) and triangulated positions $(+, \circ)$. The large variation in height is an indication for the presence calibration errors.

\section{ACKNOWLEDGMENT}

This work was partly supported by MC IMPULSE, a European Commission, FP7 research project and by the strategic research center MOVIII, funded by the Swedish Foundation for Strategic Research, SSF.

\section{REFERENCES}

[1] Time Domain, Jan. 2009. [Online]. Available: http://www. timedomain.com/

[2] Ubisense, Jan. 2009. [Online]. Available: http://www.ubisense. net/

[3] S. Sczyslo, J. Schroeder, S. Galler, and T. Kaiser, "Hybrid localization using UWB and inertial sensors," in Proc. IEEE Int. Conf. Ultra-Wideband, vol. 3, Hannover, Germany, Sep. 2008, pp. $89-92$.

[4] S. Pittet, V. Renaudin, B. Merminod, and M. Kasser, "UWB and MEMS based indoor navigation," The Journal of Navigation, vol. 61 , no. 3, pp. 369-384, Jul. 2008.

[5] M. D. Shuster, "A survey of attitude representations," The Journal of the Astronautical Sciences, vol. 41, no. 4, pp. 439517, Oct. 1993.

[6] H. L. V. Trees, Detection, Estimation, and Modulation Theory, Part I. John Wiley \& Sons, Ltd, 1968.

[7] O. J. Woodman, "An introduction to inertial navigation," University of Cambridge, Computer Laboratory, Tech. Rep. UCAMCL-TR-696, Aug. 2007.

[8] A. Chatfield, Fundamentals of High Accuracy Inertial Navigation, 3rd ed. USA: American Institute of Aeronautics and Astronautics, 1997, vol. 174.

[9] D. H. Titterton and J. L. Weston, Strapdown inertial navigation technology, ser. IEE radar, sonar, navigation and avionics series. Stevenage, UK: Peter Peregrinus Ltd., 1997.

[10] S. Gezici, Z. Tian, G. Giannakis, H. Kobayashi, A. Molisch, and Z. Poor, H.V.and Sahinoglu, "Localization via ultra-wideband radios: a look at positioning aspects for future sensor networks," IEEE Signal Process. Mag., vol. 22, no. 4, pp. 70-84, Jul. 2005.

[11] P. Misra and P. Enge, Global Positioning System: Signals, Measurements, and Performance, 2nd ed. Lincoln, MA, USA: Ganga-Jamuna Press, 2006.

[12] A. Sayed, A. Tarighat, and N. Khajehnouri, "Network-based wireless location: challenges faced in developing techniques for accurate wireless location information," IEEE Signal Process. Mag., vol. 22, no. 4, pp. 24-40, Jul. 2005.

[13] Y. Chan and K. Ho, "A simple and efficient estimator for hyperbolic location," IEEE Trans. Signal Process., vol. 42, no. 8, pp. 1905-1915, Aug. 1994.

[14] J. Nocedal and S. J. Wright, Numerical optimization. New York: Springer-Verlag, 2006.

[15] J. D. Hol, "Pose estimation and calibration algorithms for vision and inertial sensors," Lic. Thesis No 1379, Dept. Electr. Engineering., Linköping University, Sweden, May 2008.

[16] T. Kailath, A. H. Sayed, and B. Hassibi, Linear Estimation. Prentice-Hall, Inc, 2000. 